\title{
Optimization of composite structures with curved fiber trajectories
}

\author{
Etienne Lemaire ${ }^{1}$, Samih Zein $^{1}$ and Michael Bruyneel ${ }^{1,2^{*}}$ \\ ${ }^{1}$ SAMTECH s.a. (A Siemens Company), Liège Science Park, \\ Rue des Chasseurs-ardennais 8, Angleur, B-4031, Belgium \\ ${ }^{2}$ Aerospace \& Mechanical Engineering Department, University of Liège, Belgium \\ * michael.bruyneel@ulg.ac.be
}

\begin{abstract}
This paper presents a new approach to generate and optimize parallel fiber trajectories on general non planar surfaces based on level-sets and the Fast Marching Method. Starting with a (possibly curved) reference fiber direction defined on a (possibly curved) meshed surface, the new method allows defining a level-set representation of the fiber network for each ply, and so defining the fiber trajectories. This new approach is then used to solve optimization problems, in which the stiffness of the structure is maximized (minimum compliance problem). The design variables are the parameters defining the position and the shape of the reference curve. The shape of the design space is discussed, regarding local and global optimal solutions. The possibility to include in the optimization problem a limitation on the curvature of the trajectories is also addressed.
\end{abstract}

Keywords: optimization, fiber placement, level-set

\section{Introduction}

The use of composite materials in aerospace, automotive and ship industry allows manufacturing lighter and more efficient mechanical structures. Indeed, proper use of the orthotropic properties of these materials enables further tailoring of the structure to the loadings than when using isotropic materials. However, this comes at the cost of a more complicated design and sizing process firstly because of the orthotropic behavior of composite materials but also because of the manufacturing process which induces specific constraints in the use of these materials. From the mechanical point of view, one of the most important restrictions resulting from the practical manufacturing of mechanical parts is the orientation of the reinforcement fibers resulting from the layup process. These orientations directly determine the orthotropy axes and cannot be chosen arbitrarily in any point of a given part but rather result from the draping of the reinforcement material over the part. Several models have been developed in order to predict the orientations of the reinforcement fibers after the draping process depending on the properties of the reinforcement materials (see [1] for a review).

\footnotetext{
*Corresponding author: michael.bruyneel@ulg.ac.be
} 
One of the first of these models is due to Mack and Taylor [2]. Often called the 'pin-jointed' model [3], it is based on a geometric model of the woven and it is well suited to predict the fiber orientation resulting from hand layup of dry woven fabrics. Later, more complex models relying on a finite element mechanical modeling of the reinforcement have been developed for the forming of preimpregnated fabrics as for instance by Cherouat and Bourouchaki [4].

Besides the manufacturing of composites part by hand layup of large pieces of reinforcement material, another group of methods is gaining interest since its first introduction in the 1970s. These methods rely on the robotized layup of bands of unidirectional reinforcement material allowing more accurate and more repeatable manufacturing process [5]. In this group, two main methods can be identified Automated Tape Layup (ATL) and Automated Fiber Placement (AFP). ATL makes use of a robotic arm to layup tapes (up to $300 \mathrm{~mm}$ wide) of unidirectional prepeg and benefits from high productivity for large and simple flat parts. But ATL main limitation comes from the relatively high minimum curvature radius (up to $6 \mathrm{~m}$ ) that can be applied to the prepreg tape without wrinkling. With AFP, this minimum curvature radius is decreased to $50 \mathrm{~cm}$ by subdividing the tape into several tows which can be cut and restarted individually. Therefore the manufacturing of more complicated parts can be handled by AFP but with a lower productivity than ATL.

For ATL and AFP processes, one of the manufacturing issues is the determination of successive courses trajectories. Indeed, for these processes, it is crucial that there are no overlaps and no gaps between adjacent courses in order to ensure maximal strength and minimum weight for the final part. In other words, this means that successive layup courses have to be equidistant.

A few researchers have studied the optimal design of ATL/AFP parts. A first group of methods consists in defining an initial course which is then simply shifted over the part to define subsequent course as proposed by Tatting and Gürdal $[6,7]$. Secondly, the courses can be defined as geodesic paths, constant angle paths, linearly varying angle paths or constant curvature path [8]. However, these two approaches do not result in equidistant paths. Alternatively, the subsequent courses can be obtained by computing actual offset curves from an initial curve. This approach is more difficult but leads to equidistant courses and has been investigated by Waldhart [9], Shirinzadeh et al [10] and Bruyneel and Zein [11] with different numerical schemes. The two first groups of authors propose an approach based on a geometrical description of the part while the third one developed an algorithm able to work with a level-set representation on a mesh of the layup surface. In [12], Bruyneel and Zein used their approach in a parametric study in order to determine the effect on the structural stiffness of curving the fiber trajectory of a single ply laid on a cantilever structure (see also [13]). It is also worth mentioning the work done recently by Brampton and Kim [14] where level-set and optimization approaches are used on planar structures.

The goal of the present paper is to demonstrate further the capabilities of the method proposed by Bruyneel and Zein [11], which is available in an industrial context, by using it for optimal design of composite parts. 
This paper starts with a brief introduction describing the method developed in $[11,12]$ to determine equidistant courses for ATL/AFP process. Next, several optimization problems with growing complexity are studied in order to illustrate the interest of the method.

\section{Fiber placement modeling}

\subsection{Fast Marching Method}

Bruyneel and Zein [11,12] first proposed the use of level-set and Fast Marching Method (FMM, see [15]) to solve the problem of determining equidistant courses on an arbitrary layup surface. The Fast Marching Method aims at solving the Eikonal equation:

$$
\begin{gathered}
|\nabla T(x)|=f, \quad x \in \Omega \backslash \Gamma, \\
T(x)=0, \quad x \in \Gamma C \Omega .
\end{gathered}
$$

The problem given in Eq. (1) consists in finding a scalar field $T(x)$ such that the norm of its gradient is constant over the domain $\Omega$ and that the value of $T$ is equal to zero on a curve $\Gamma$ of $\Omega$.

As illustrated in Fig. 1, this differential equation can be interpreted as the one characterizing a front propagation at a constant speed where $T(x)$ denotes the time at which the front passes through point $x$. At time $T=0$, the front coincides with the curve $\Gamma$, therefore, all points located on the curve $\Gamma$ will have a value of $T$ equal to 0 . Then as time increases, the front propagates at a constant speed equal to $1 / f$ over $\Omega$. The position of the front $\Gamma_{1}$ at any time $T_{1}$ corresponds to the set of point lying on the isovalue $T(x)=T_{1}$. Since the front speed norm is uniform over the domain, every point of $\Gamma_{1}$ is equidistant from $\Gamma$. The set of equidistant curve can therefore be obtained by selecting appropriate isovalues of $T(x)$ over $\Omega$. 


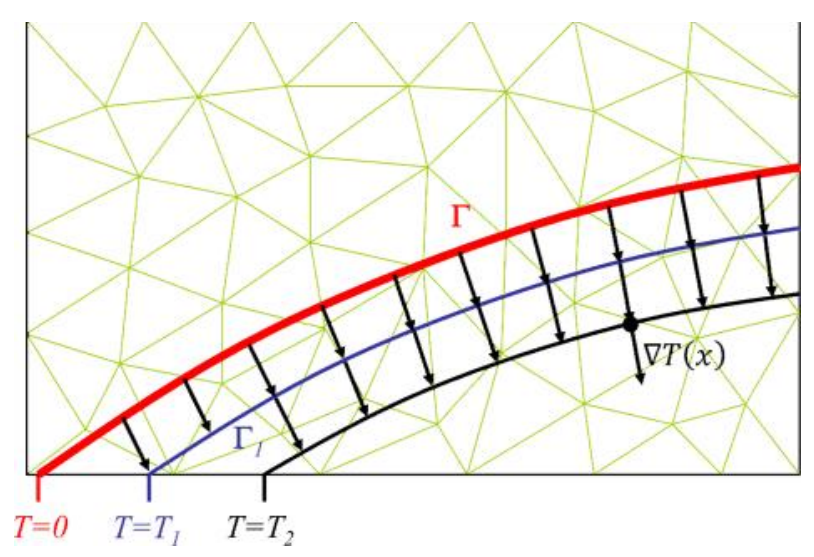

Figure 1. Front propagation interpretation of Eikonal equation.

Based on a triangular mesh of the layup surface, the developed procedure allows computing fiber orientation on each element of the mesh. At first one needs to define the initial front position on the layup surface. This curve corresponds to the reference course and the definition procedure is presented in next subsection. Secondly, the Fast Marching Method is used to solve the Eikonal equation and to compute the time $T$ at any point of the mesh. The function $T(x)$ is supposed to be piecewise linear by element. Starting from initial values defined by the reference curve, the value of $T$ is progressively computed on the domain by solving the Eikonal equation locally on each triangle of the mesh. For further details about the Fast Marching Method, the interested reader may refer to [15]. Finally, the fiber orientations on each element are defined by computing the direction of the isovalues of $T(x)$ over the considered element. Since those isovalues are equidistant from the reference course, the computed orientations correspond to a gap-less and overlap-less (i.e. constant thickness) layup obtained by ATL or AFP.

\subsection{Reference course tracing}

The definition of the reference course plays a major role in the context of the present work since the orientation or the control points of the corresponding curve are used as design parameter of the optimization problem.

Because the definition of a curve on a general 3D surface may be a difficult task, we have chosen to resort to an 'artificial' 2D space to define the reference curve and next to map this curve onto the layup surface to obtain the reference course. This process is illustrated in Fig. 2. The reference curve is defined in the 2D space such that it passes through the axes origin. A seed point is defined on the $3 \mathrm{D}$ surface and the triangle containing this point is mapped in the 2D 'artificial' space such that the seed point lies on the origin. The intersection between the reference curve and the mapped triangle is approximated by a line segment which is transposed to the 3D space to define the first segment of the reference course. Next, this process is repeated for the next triangle that is intersected by the initial course (e.g. the triangle adjacent to edge AC in Fig. 2) until the boundary of the $3 \mathrm{D}$ surface is met. At the end of this process the initial course is obtained on the layup surface mesh as a piecewise linear curve. 


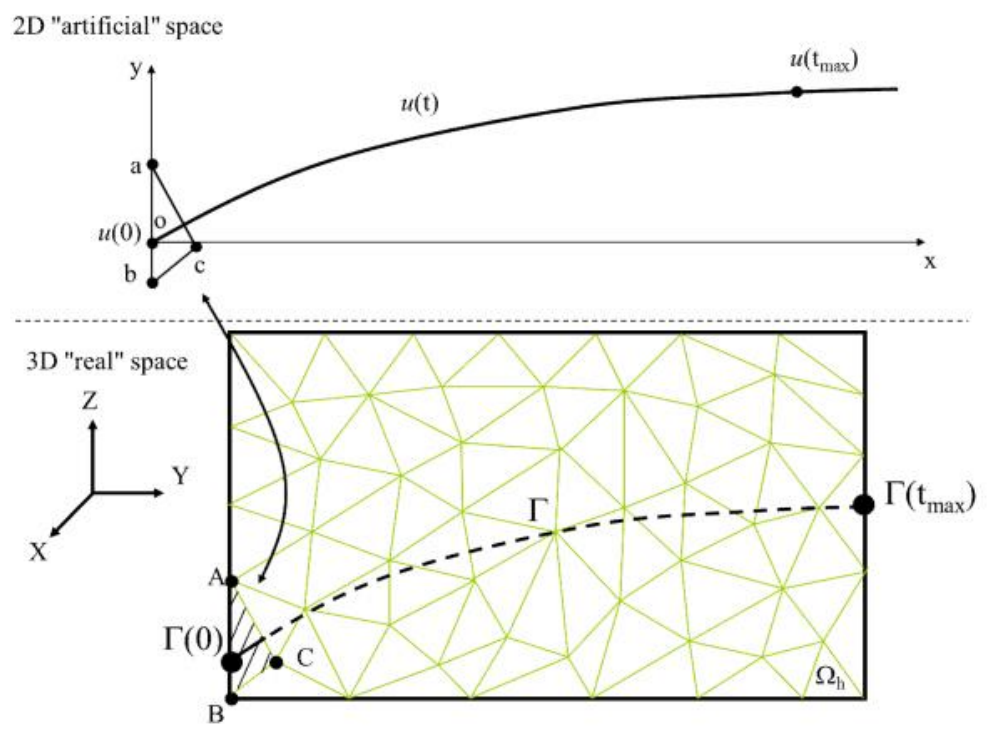

Figure 2. Reference course mapping.

\section{Straight course optimization}

\subsection{Optimization problem}

The first illustration of the method is a very simple optimization problem consisting in minimizing the compliance (maximizing the stiffness) deformationenergy of a square plate presented in Fig. 3. The left side of the plate is clamped and a point force is applied downwards at the lower right corner. For more convenience and since it is possible for the present 2D structure, the reference curve is presented directly on the structure (even if the mapping process described in previous section is used). The initial course is supposed to be straight and the sole design variable of the problem is $\theta$ the angle made by this course and the horizontal. The seed point is fixed at the half of the left side boundary of the plate. 


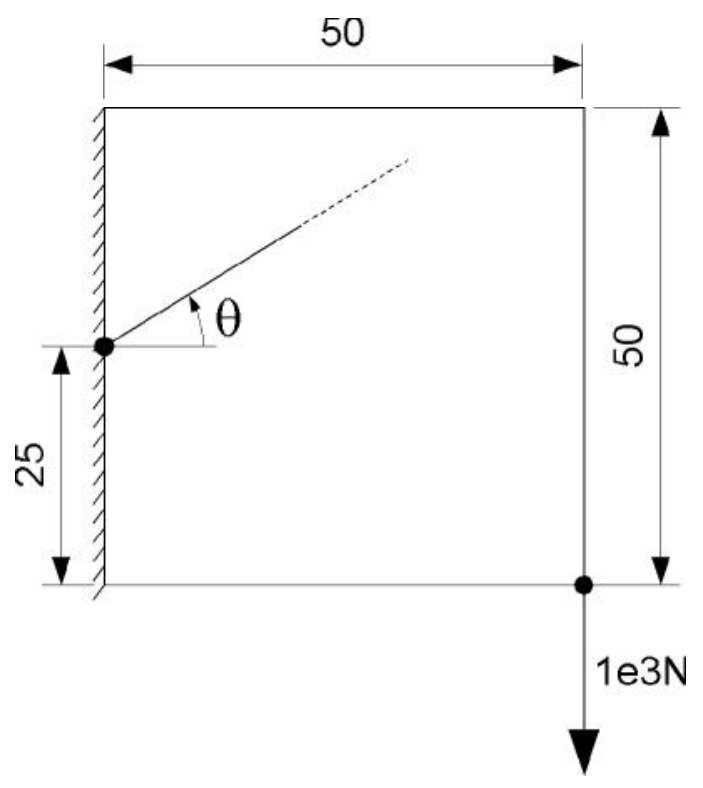

Figure 3. Straight fiber path optimization problem.

For the present application, layup courses are obviously very simple as the planar geometry of the structure and the choice of a rectilinear initial course always results in uniform fiber orientations over the structure. However, the purpose of this application is to validate the optimization approach and also to provide a reference solution for further examples.

The optimization problem is mathematically formulated in Eq. (2) where $c$ stands for the deformation energy (compliance). Upper and lower bounds and a lower are imposed on the angle such that all possible orientations are covered.

$$
\begin{gathered}
\min _{\theta} c(\theta), \\
\text { s.t. }\left\{-90^{\circ} \leq \theta \leq 90^{\circ} .\right.
\end{gathered}
$$

The material properties used for all numerical applications are those of a typical composite used for ATP made of unidirectional carbon fibers. Classical carbon/epoxy properties are used.

\subsection{Solution}

The numerical model is created by meshing the plate with 40x40 membrane elements. The optimization is carried out using the optimizers available in the BOSS Quattro optimization tool box [16]. Gradient based optimization [17] will be used for this application. The required sensitivities can be computed using finite difference since due to the small number of design variable it does not lead to a prohibitive computational overhead.

To initiate the optimization process a course perpendicular to the left edge (i.e. $\theta=0^{\circ}$ ) is selected which gives an initial value of the objective function equal to $11.919 \mathrm{~J}$. After a few iterations, the optimization method converges to an optimum for $\theta=-42.9^{\circ}$ and $c=8.718 \mathrm{~J}$ which means a decrease of $27 \%$ with respect 
to the initial value. The resulting fiber orientations and courses are presented in Fig. 4. In this figure, the red line represents the initial course centerline and each color strip corresponds to a layup course. Resulting elementary fiber orientations are represented by a small segment on each element.

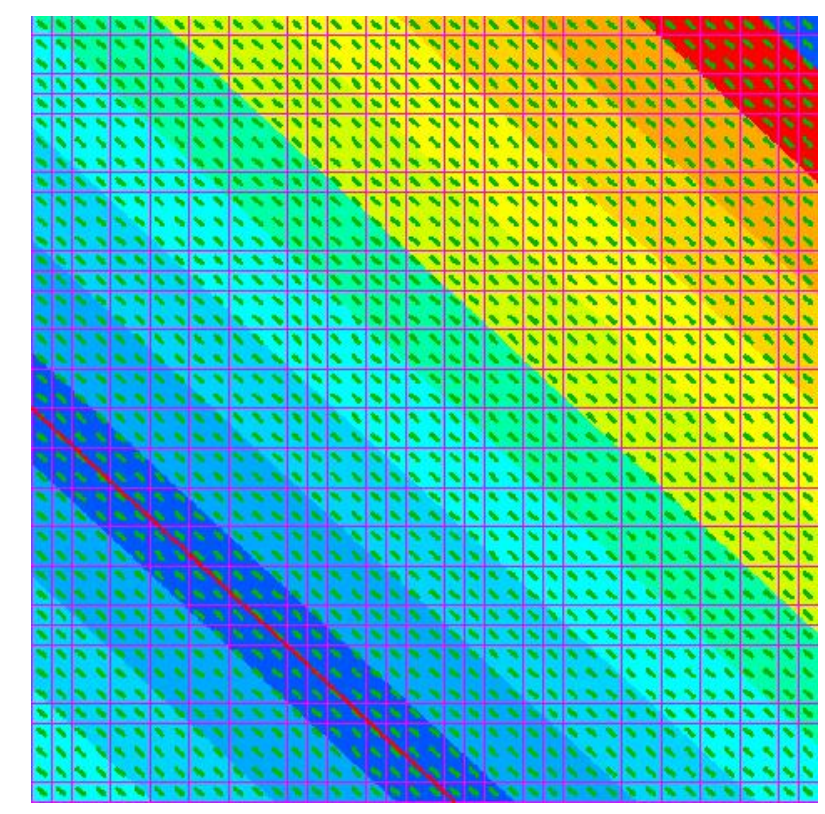

Figure 4. Solution of the optimization problem with a straight course.

Since the optimization problem considered in this section is very simple the validity of the optimal solution presented in Fig. 4 can be verified by performing a parametric study over the design domain.

Therefore, the value of the objective function has been computed for every integer value of $\theta$ between $-90^{\circ}$ and $90^{\circ}$. The result of the parametric study is presented in Fig. 5. The minimal value of the objective function is $8.719 \mathrm{~J}$ obtained for $\theta=-43^{\circ}$ which confirms the result of the optimization procedure. Moreover, the parametric study shows that the present optimization problem does not possess local optima except the one generated by the upper bound $\theta<90^{\circ}$. However, as the objective function is periodic, the solution $\theta=90^{\circ}$ would disappear if the upper bound was larger and lead to the same solution as $\theta=-43^{\circ}$. 


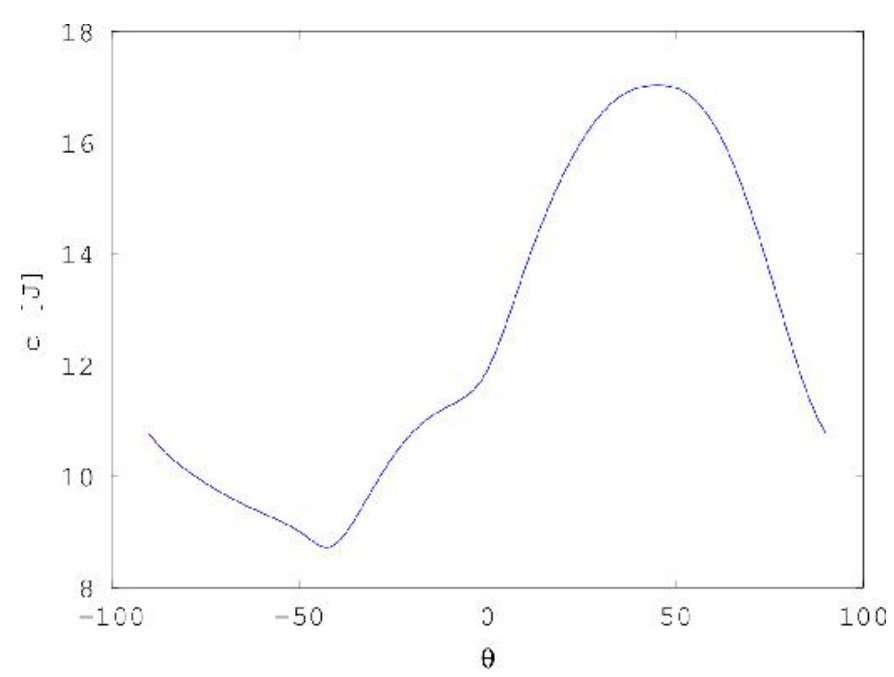

Figure 5. Deformation energy versus the angle design variable.

\section{Curvilinear course optimization}

\subsection{Optimization problem}

In order to make advantage of fiber placement capabilities, we can consider a more general optimization problem with a curvilinear fiber course presented in Fig. 6. In this new optimization problem, the geometry of the structure and the boundary conditions remain identical to the one considered in previous section while the definition of the initial course is modified. Firstly, the optimization process is now able to move the seed point along the left side of the plate by adjusting the design variable $y_{s}$. Secondly, the initial course corresponds now to a quadratic spline defined by three points: 1/ The seed point: 2/ A final point located on the right edge at a vertical distance from the seed point equal to $y_{p}$ (the second design variable); 3 / A middle point placed at $25 \mathrm{~mm}$ from the left edge and at a vertical distance from the seed point equal to $y_{p} / 5$.

The mathematical formulation of the optimization problem is given in Eq. (3). Again, the objective of the optimization problem is to minimize the deformation energy.

$$
\begin{gathered}
\min _{y_{p}, y_{s}} c\left(y_{p}, y_{s}\right), \\
\text { s.t. }\left\{\begin{array}{c}
1 \leq y_{s} \leq 49, \\
-100 \leq y_{p} \leq 100 .
\end{array}\right.
\end{gathered}
$$

The position of the seed point $y_{s}$ is restricted to the range $[1,49]$ in order to avoid discontinuities of the objective function that may arise when the seed point is close to the lower or upper edge of the plate. Indeed, when the seed point is close to one of those edges, the initial course may suddenly go out of the domain for a small modification of $y_{s}$, which leads to very different layups. 


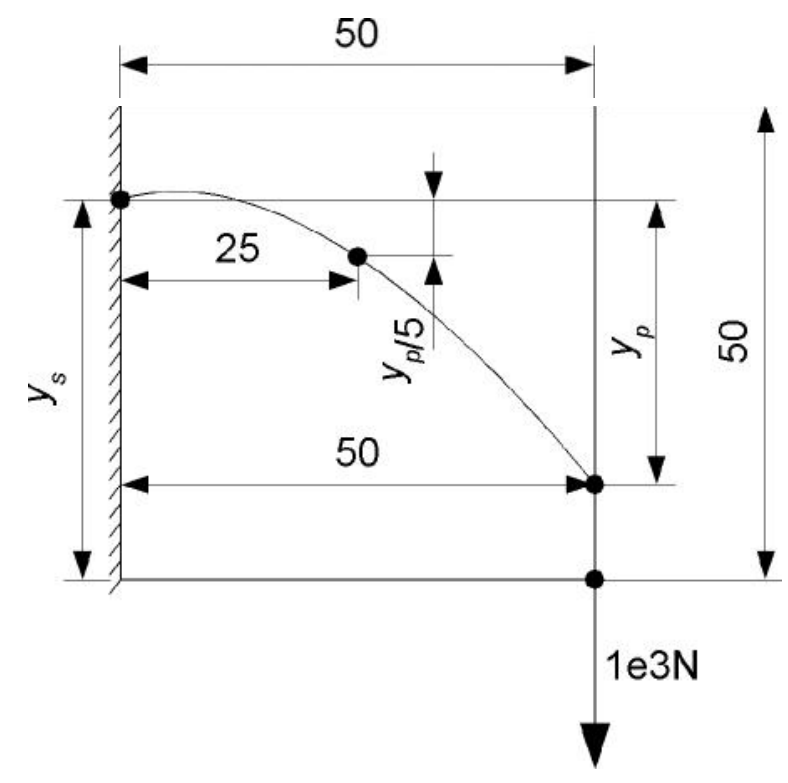

Figure 6. Curved fiber path optimization problem.

\subsection{Solution}

The optimization problem is solved with the same procedure as previously based on finite difference sensitivities. The initial design is chosen as in previous application with $y_{s}=25$ and $y_{p}=0$ which result in horizontal rectilinear fibers. In the final design presented in Fig. 7, the position of the seed point has reached its upper bound $\left(y_{s}=49\right)$ while the end point of the initial course is defined by $y_{p}=-$ 48.7 .

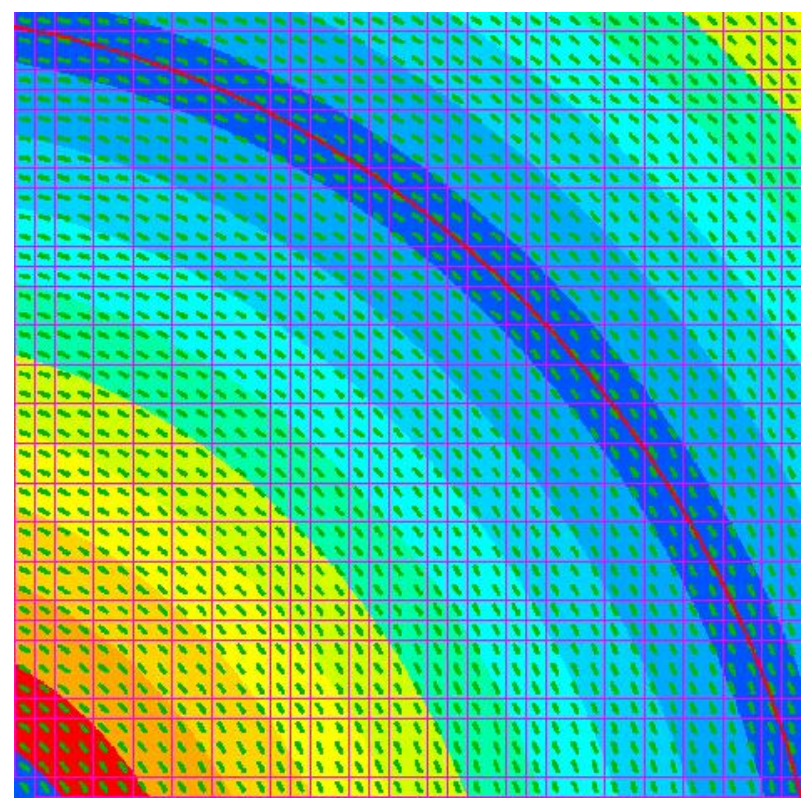

Figure 7. Solution of the optimization problem with a curvilinear course. 
Under the design load case, the final design gives a deformation energy which is $61 \%$ less than the initial design and $46 \%$ less than the rectilinear design obtained previously. This shows that the capability of AFP to follow curved courses leads to a strong improvement of the mechanical performance and confirms the conclusions of Hyer and Charette [18]. Nevertheless, we can observe that the curvature of the courses increases in the lower left corner such that the introduction of an optimization constraint on curvature would be helpful to ensure the manufacturability of the part. This issue will be addressed in Section 6 .

Figure 8 illustrates the iteration history of the optimization problem. The performance of a gradient-based method, an adaptive surrogate approach and a genetic algorithm, is compared. Details of these optimizers are available in [17]. The default values of the optimizers were used [16]. The gradient-based optimizer needs 17 iterations to converge. Since finite differences are used for the computation of the function derivatives (sensitivity analysis), 51 function evaluations are required as 2 design variables are defined in the problem. The adaptive surrogate method and the genetic algorithm need 89 and 171 function evaluations, respectively, to reach the optimum. The optimal values of the objective function and of the design variables provided by the three methods are very similar, and the resulting optimal objective functions only differ from less than $1 \%$. It is observed that the genetic algorithm provides the worst solution (higher value of the objective function), certainly because the default values of the algorithm should have been adapted to the problem. Anyway, the three solutions are very close.

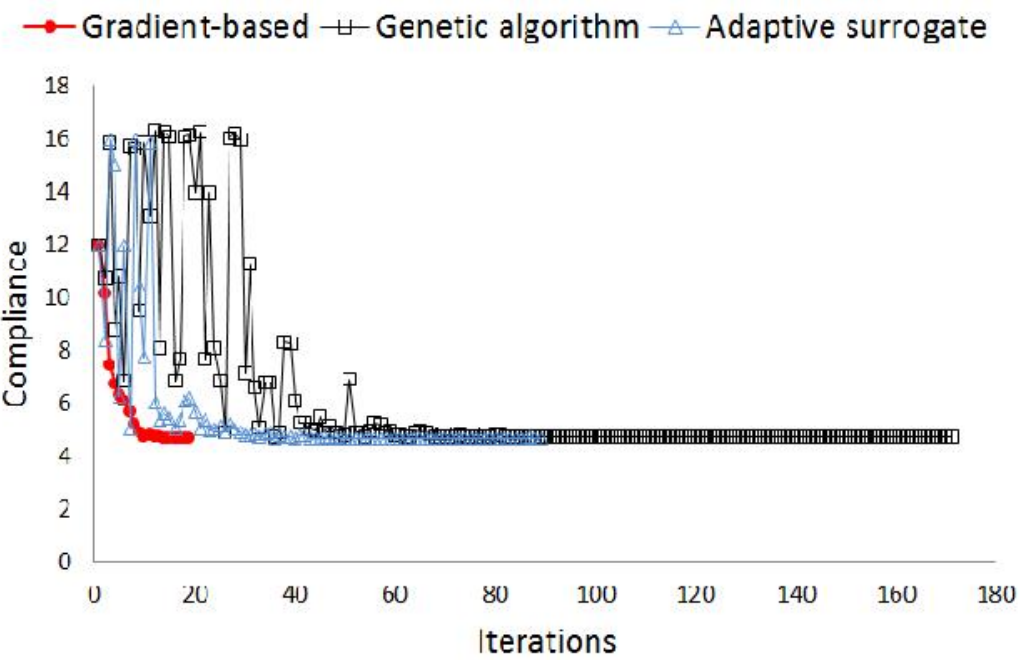

Figure 8. Iteration history for the solution of the optimization problem with a curvilinear course (relative compliances)

In order to check the optimality of the design obtained by the optimization procedure and to investigate the existence of local optima, a parametric study has been performed. The result of this parametric study is presented in Fig. 9. The plotted surface represents the objective function value over the optimization domain. We can check that the presented design actually corresponds to the 
optimal solutions. However, a closer analysis of Fig. 9 shows that there is also a local optimum for $y_{s}=1$ and $y_{p}=62$, corresponding to a large value of the objective function, into which the optimization procedure based on the gradient could get trapped if a different initial design is selected.

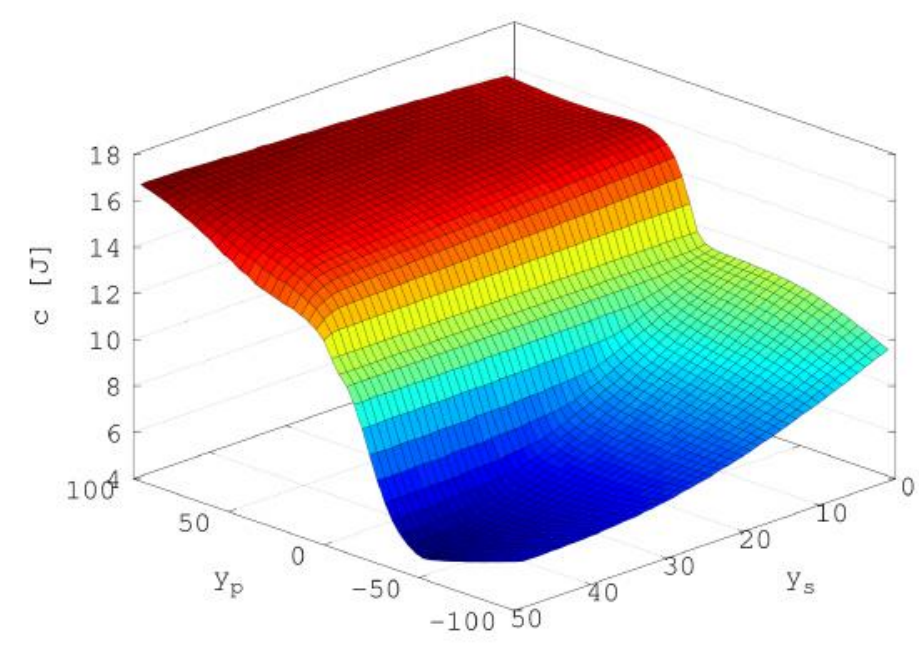

Figure 9. Objective function for the curvilinear course optimization problem

\section{Conical surface}

\subsection{Optimization problem}

In order to illustrate the ability of the method to handle 3D surfaces, the next presented numerical application is the optimal design of a conical shell depicted in Fig.10. The cone axis coincides with $\mathrm{Z}$ axis. The large base of the cone is clamped while a force and a torsion torque are applied on the small base. The shell is composed of two plies which can be oriented independently. For each ply, the reference curve is a straight line in the 'artificial' 2D plane and its angle with respect to the $\mathrm{x}$ axis of the 'artificial' $2 \mathrm{D}$ plane is a design variable. We have therefore two design variables $\theta_{1}$ and $\theta_{2}$. The mathematical formulation of the optimization problem is:

$$
\begin{gathered}
\min _{\theta_{1}, \theta_{2}} c\left(\theta_{1}, \theta_{2}\right), \\
\text { s.t. }\left\{\begin{array}{l}
-90^{\circ} \leq \theta_{1} \leq 90^{\circ}, \\
-90^{\circ} \leq \theta_{2} \leq 90^{\circ} .
\end{array}\right.
\end{gathered}
$$

Moreover, because the surface is conical, if the reference course makes more than one revolution around the cone, it becomes non equidistant to itself. As a consequence it is of course impossible to generate equidistant courses. To circumvent this problem, we assume that the shell is manufactured in two parts which are draped with the same parameters. That's why in Fig. 10, one seed point is placed on each side of the (YZ) plane and the shell is cut in two by this plane. 

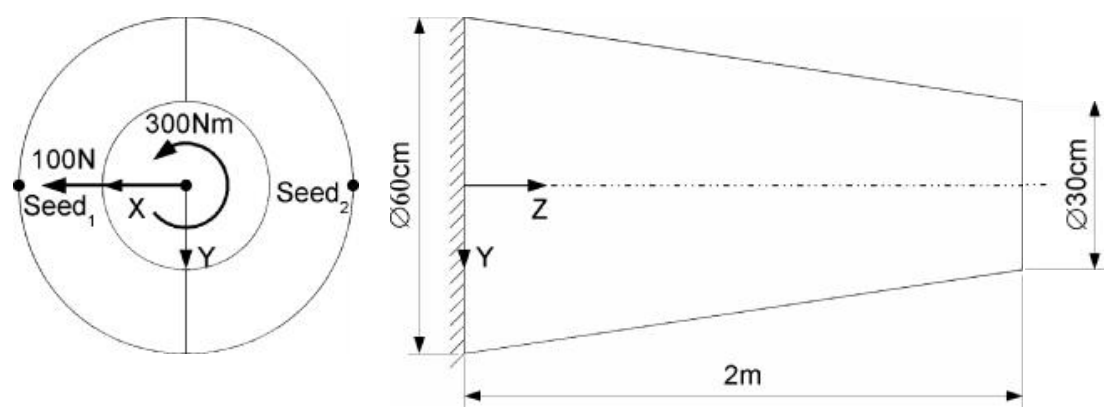

Figure 10. Conical surface dimensions and load case.

\subsection{Solution}

Starting with $\theta_{1}=\theta_{2}=0$, the optimization procedure converges to the solution presented in Fig. 11. Ply 2 is the symmetrical of ply 1 with respect to plane (X-Z) as at the end of the optimization process $\theta_{1}=37.7^{\circ}$ and $\theta_{2}=-37.7^{\circ}$. These

opposed orientations shows that in the present load case, torsion dominates. The deformation energy is cut by $83 \%$ between the initial design and the final design.

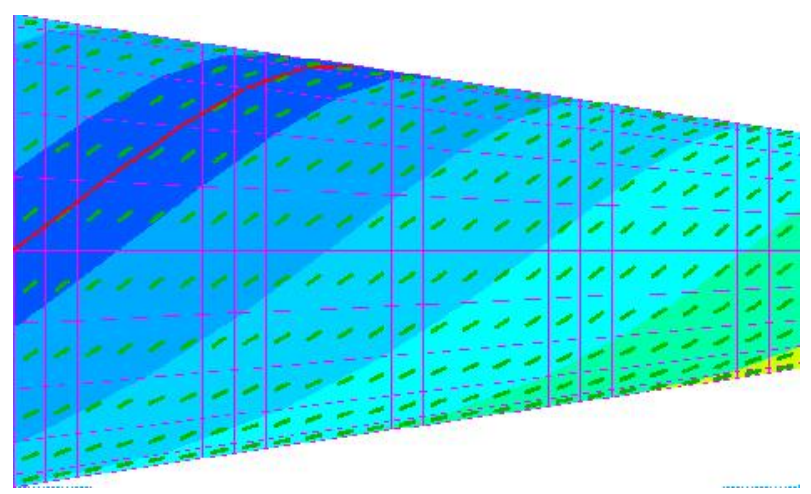

a. Ply 1

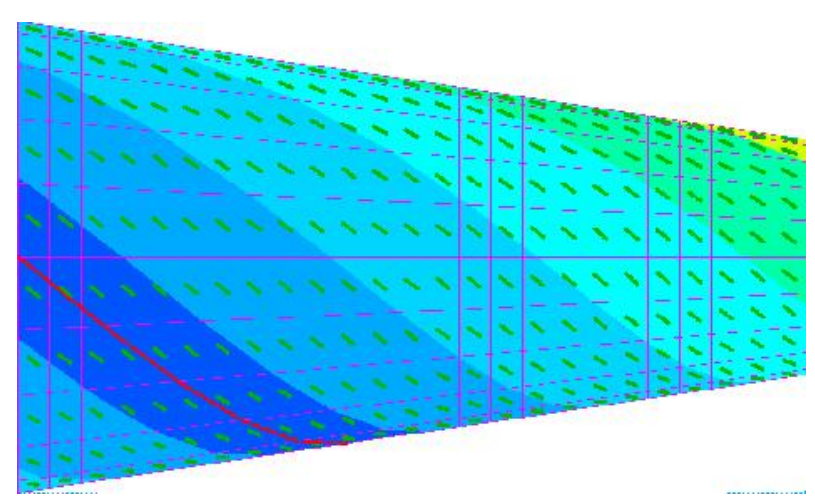

b. Ply 2.

Figure 11. Solution of the conical shell optimization problem. 
The results of a parametric study are presented in Fig. 12 which presents the isovalue lines of the objective function over the design space. One can notice that the objective function has several local extrema but fortunately, only two of them are minima. The solution obtained by the optimization procedure is indicated by a star label and actually corresponds to a minimum of the objective function. The second minimum corresponds in fact to the first one but with changed sign for each design variable. As a consequence, we can conclude that the stacking sequence has no significant importance for the present application.

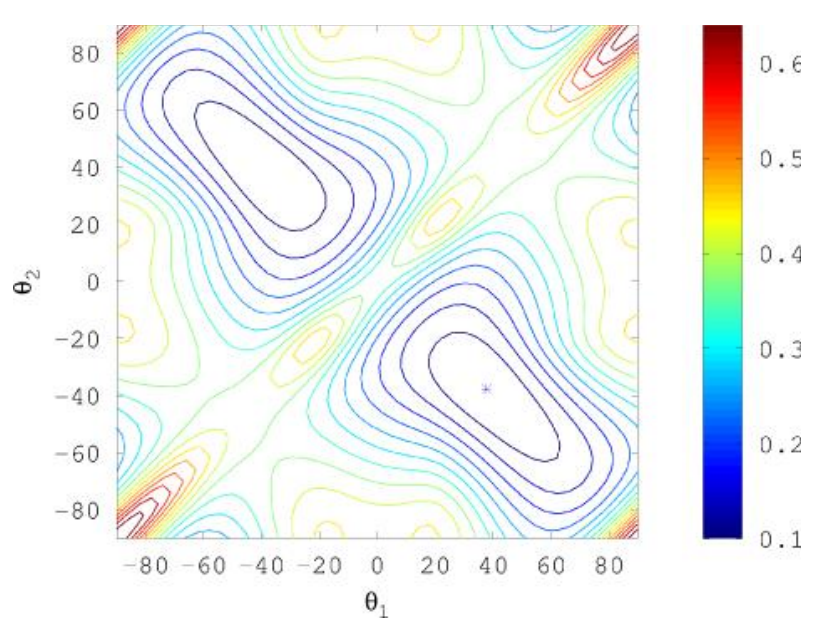

Figure 12. Objective function isolines over the design space.

\section{Constraint on the curvature}

\subsection{Curvature computation}

As mentioned previously, the solution reported in Figure 7 shows high curvatures at the bottom left corner. Depending on the fibre placement machine and on the material, such trajectories presenting a high curvature may be not allowed or produce a design that can't be manufactured. In this section, we introduce constraints on the trajectory curvature in the optimization problem.

The curvature of a curve lying on a 3D surface can be subdivided in two components; the normal and the geodesic curvature [19]. The normal curvature $\kappa_{n}$ corresponds to the curvature which is imposed by the surface to the curve. It is equal to the curvature of the surface along the direction of the tangent to the curve. For instance for a planar surface it is always zero, for a sphere it is always equal to the inverse of the radius of the sphere. The geodesic curvature $\kappa_{g}$ corresponds to the proper curvature of the curve inside the surface. It can be seen as the remaining curvature obtained after projecting the curve on the surface tangent plane. The total curvature of the curve $\kappa$ (its curvature in the $3 \mathrm{D}$ space) is related to the geodesic and normal curvatures by the following equation:

$$
\kappa^{2}=\kappa_{n}^{2}+\kappa_{g}^{2}
$$


From an ATP/AFP manufacturing point of view, the geodesic curvature determines the in plane bending applied to the fiber tape or tow. This is the direction in which the tapes and the tows are the less flexible and attempting to impose a too low turning radius may lead to defects. This is why we focus here on evaluating the geodesic curvature of the fibers.

Once the distance field to the initial layup course is obtained using the Fast Marching Method, the subsequent layup courses and fiber path can be obtained by extracting some isovalues from the distance field. Consequently, these isovalues can be used to compute the curvature of the fibers. Therefore, the fiber curvature is evaluated over the surface by tracing a levelset portion passing through every node of the mesh as illustrated for node $i$ in Figure 13. Next, in order to cancel the normal curvature, the strip of triangles supporting the levelset portion is unfolded on a plane by progressively rotating each triangle around its intersection with the previous one. This method is derived from the one proposed by Mitchell et al [20] to trace geodesic curves on meshed surfaces.

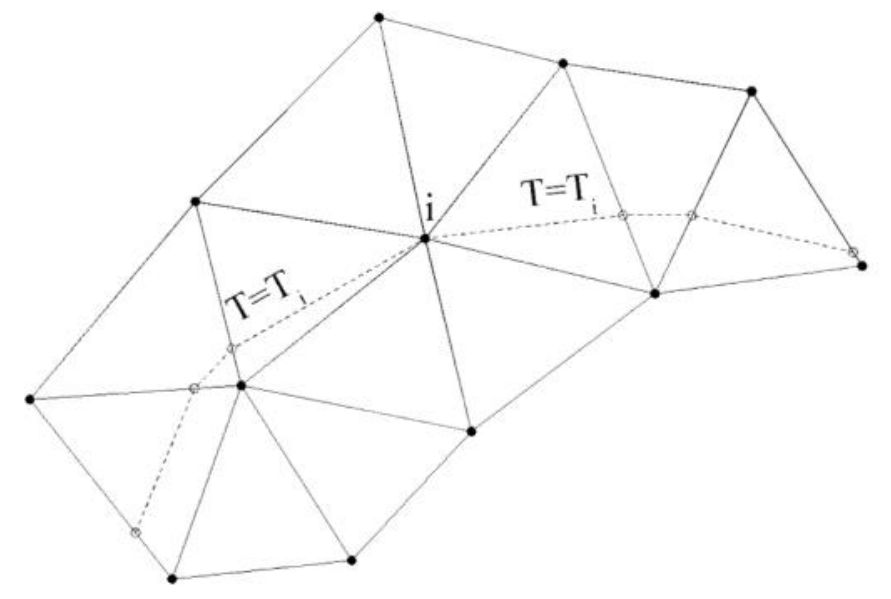

Figure 13: Discrete level-set passing through node i.

However because of the basic assumptions of the Fast Marching Method the obtained curves are discrete curves made of line segments which link successive vertices. The definition of the curvature of a discrete curve is not straightforward. Indeed if one applies the classical definition of the curvature for smooth curves to discrete curves, it leads to a zero value along the segment and sudden infinite value at vertices (if successive segments are not collinear). As explained by Coeurjolly et al. [21], two possibilities exist to compute local curvature of discrete curves. Firstly, one can interpolate the vertices using parametric curves (Splines, B-Splines, ...) and use the continuous curvature definition. Secondly one can also resort to discrete curvature definitions that are available in literature.

After investigation of the two methods suggested by Coeurjolly, we came to the conclusion that the first method based on parametric curves provides the best results for our problems. The developed approach to evaluate the curvature at one node consists in using levelset portions made of 5 vertices passing the considered node. The resulting discrete curve is then fitted using a polynomial parametric curve of the third degree which curvature can then be computed analytically. The main advantage of the parametric curve fitting is that it allows considering longer levelset portions (i.e. with more vertices) such that local errors due to Fast 
Marching Approximation are more efficiently smoothed out than when resorting to discrete curvature definition which only allows considering levelset portions made of three vertices. The method has been tested on a spherical benchmark for which geodesic curvature can be computed analytically and shows to generally provide a very good estimation of geodesic curvature excepted on the boundary of the surface due to the fact that it is not possible to trace a levelset portion on both side of the considered nodes. For this reason, we decided to discard the boundary nodes from the curvature computation. Indeed, as we will see in the results, even if the maximum curvature values usually appear close to the boundary, the curvature distribution is sufficiently smooth to avoid a too large error.

\subsection{Illustration}

To illustrate the possibility to introduce a curvature constraint into the optimization problem let us consider once again the optimization problem of the square plate with a curvilinear initial layup course (Figure 7). If we evaluate the local fiber curvature for design obtained previously, we get the curvature distribution presented in Figure 14. Since the center of curvature of the initial layup course is located close to the lower left corner of the plate, the curvature increases progressively towards this corner. Consequently, the maximal curvature (0.067) is reached in the lower left corner of the plate. In order to show the capabilities of the optimization procedure, we propose to add a curvature constraint to the optimization problem in order to make sure that the maximal fiber curvature of the final design does not exceed 0.02. Like the compliance sensitivity, the sensitivities of the local curvature are computed using finite differences.

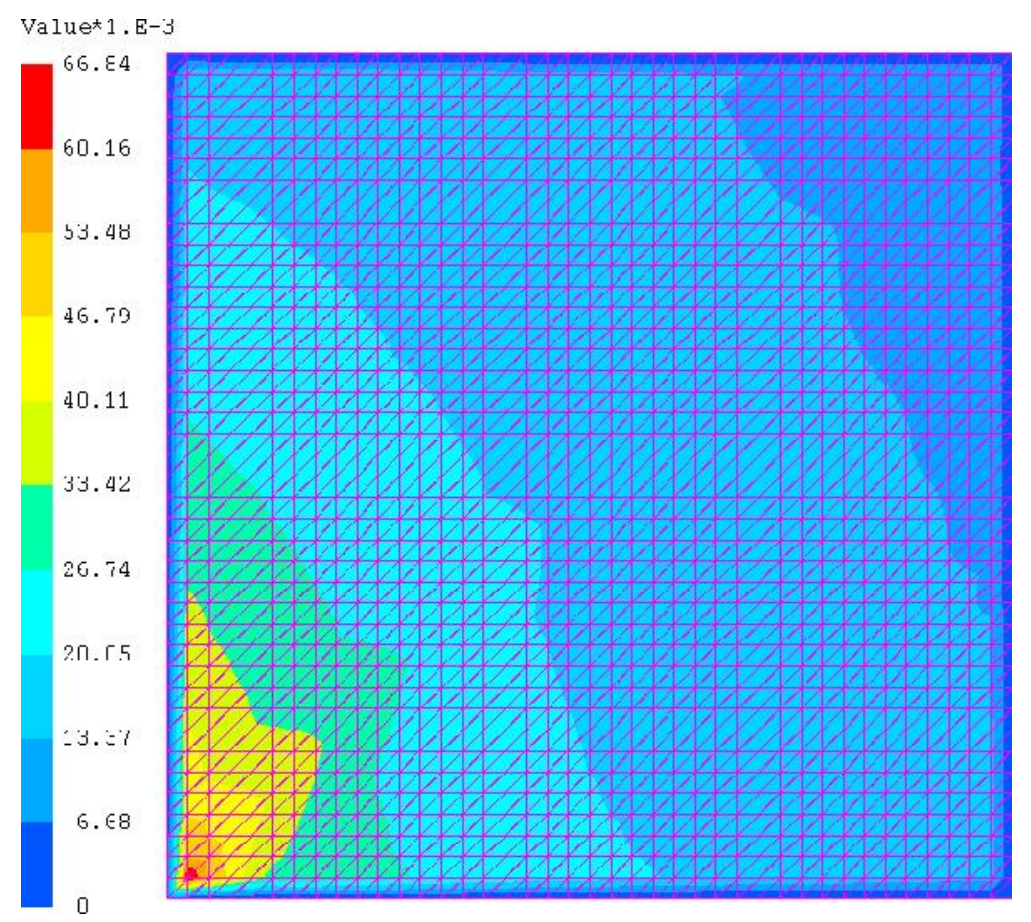

Figure 14: Resulting curvature for the compliance optimization problem.

The optimization problem presented in Eq (3) is modified by adding a constraint on all the nodal curvatures $\kappa_{\mathrm{i}}$ (i being the nodes indices): 


$$
\begin{gathered}
\operatorname{minc}\left(y_{p}, y_{s}\right), \\
\text { s.t. }\left\{\begin{array}{c}
\kappa_{i} \leq 0.02 \quad \forall \mathrm{i}, \\
1 \leq y_{s} \leq 49, \\
-100 \leq y_{p} \leq 100 .
\end{array}\right.
\end{gathered}
$$

Starting from the previous design $\left(y_{s}=49, y_{p}=-48.9\right)$ which is now non-admissible, the gradient-based optimizer is able to find a new feasible solution after 63 iterations. The new design is presented in Figure 15 and the corresponding local curvature map in Figure 16. Because of the curvature constraint, the design is different from the previous one as we get $y_{s}=47.6$ and $y_{p}=-88.1$ which leads to a straighter initial course. The final compliance is equal to $5.679 \mathrm{~J}$ which is still better than the initial value $(11.919 \mathrm{~J})$ and than the design using a straight course ( $8.718 \mathrm{~J}$ ) but $21 \%$ higher than the unconstrained design. Figure 16 shows that the upper bound on curvature is active for the new design and even if the maximum curvature is not anymore exactly located at the lower left corner of the plate, it is still in that region that the highest curvature value appears.

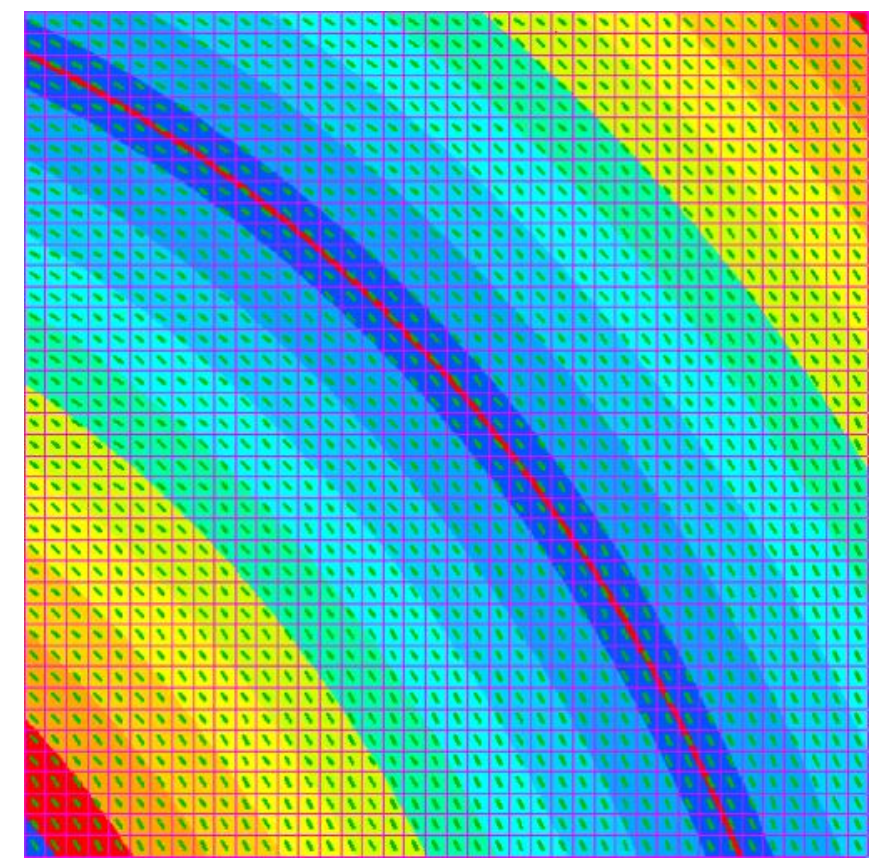

Figure 15: Solution of the optimization problem with a curvature constraint. 


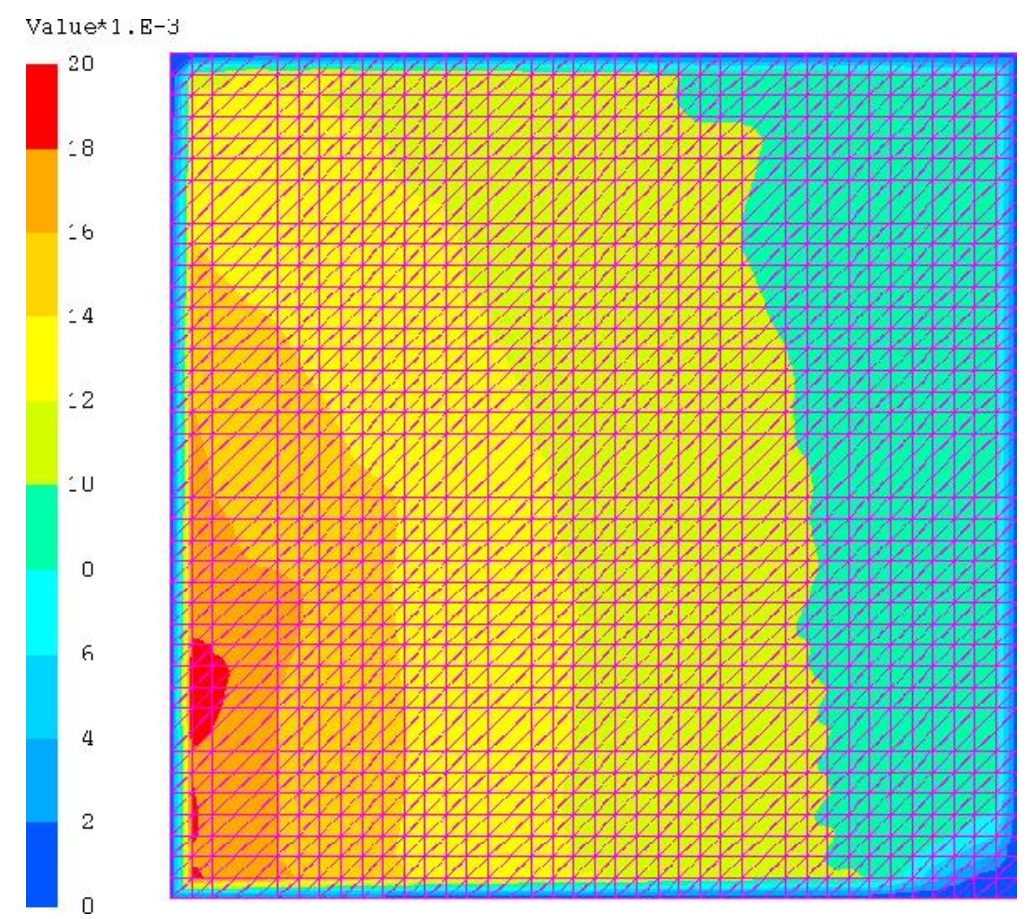

Figure 16: Fiber curvature obtained with the curvature constrained optimization problem.

A parametric study has been performed in order to analyse the characteristics of the constrained problem. Since it would not be convenient to consider all nodal curvature constraint individually, we choose to represent the maximum curvature value over all the nodes in order to visualize the feasible domain. The maximum curvature function is presented in Figure 17 as a function of the two design parameters. It presents two peaks, one of them is very close to the unconstrained optimum. Also, we can check that the maximum curvature is zero when $y_{p}=0$ which is consistent since in that case, the initial course is straight.

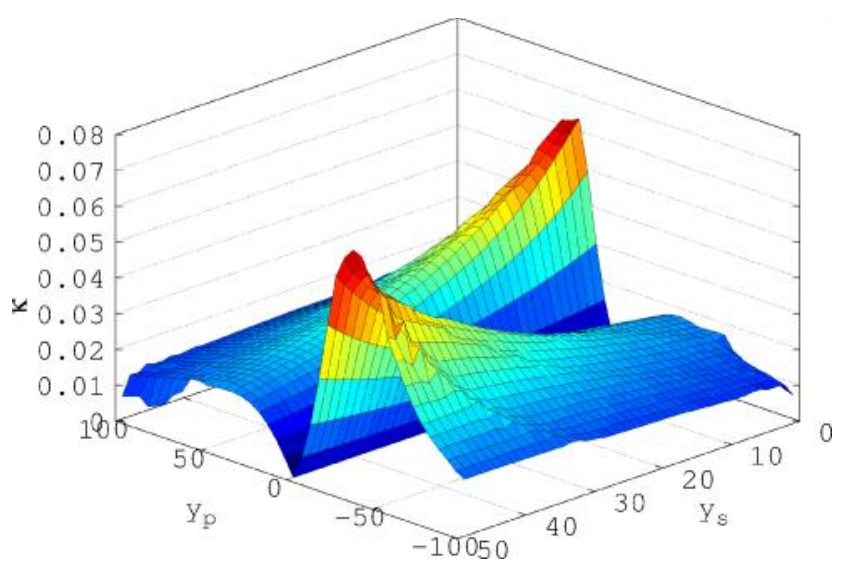

Figure 17: Maximum curvature function.

Figure 18 presents the contour plot of the compliance objective function overlaid on the feasible domain obtained by plotting the maximum curvature levelset equal to 0.02 . The non-admissible domain corresponds to the two gray areas while the white one is admissible. The constrained optimum obtained with the optimization 
procedure is marked by a blue + and is located on the constraint. Conversely, the previous design obtained given by the unconstrained optimization problem is indicated by a red $\mathrm{X}$ and is located in the non-admissible domain.

The parametric study also shows that the introduction of the curvature constraint makes the optimization problem more complicated. Firstly, we can notice that the introduction of the constraint almost leads to a partitioning of the design domain. If the maximum curvature upper bound is set to a lower value, the design domain could be split into several subdomains. The existence of isolated subdomains is a problem for gradient based optimization since classical methods are not able to move from one subdomain to another. Moreover, we observed that the maximum curvature function is slightly non-smooth which sometimes causes the optimizer to be stuck in a local optimum when the constraint becomes active.

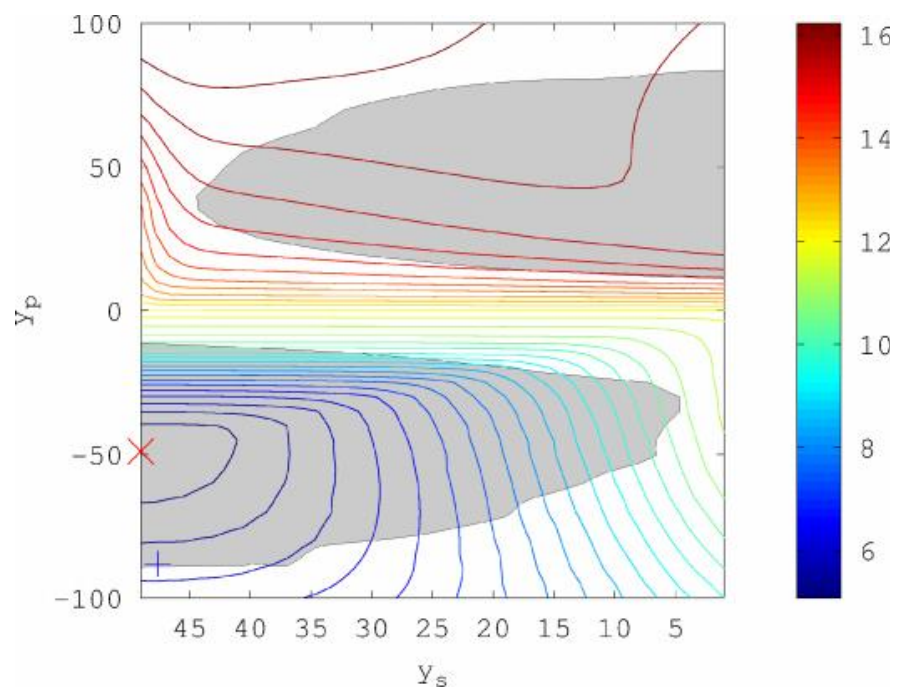

Figure 18: Design domain of the constrained optimization porblem.

\section{Conclusion}

In this paper, a new approach for computing fiber orientations resulting from ATL/AFP draping is used as the basis for composite shell optimization. The main advantages of this approach over previous research is that it allows determining fiber orientations and deposition courses that correspond to a layup free from overlaps and gaps between successive courses. Moreover, the method does not require a geometrical representation of the layup surface but can simply be applied on a 2D mesh of this surface.

In order to show the generality of the proposed approach, several numerical applications were proposed, firstly on 2D surfaces, next on a 3D surface.

Additionally, the results of these applications confirms the benefits of using curved fiber paths on mechanical performance as previously observed [18] with the great advantage in the present case that optimal fiber orientation correspond to a manufacturable part. 
Finally, a constraint on a minimum curvature radius for the layup courses was defined and introduced in the optimization problem.

As developed in commercial pieces of software, the solution is available for industrial applications. However, future work should, for instance, focus on the improvement of the sensitivity analysis. The development of a semi-analytical sensitivity analysis would strongly improve the efficiency of the optimization process and would allow increasing the number of design variables in order to consider more complex courses definition.

\section{Acknowledgement}

Part of this work was supported by Wallonia (Belgium), in the DRAPOPT project.

\section{References}

1. T.-C. Lim and S. Ramakrishna, "Modelling of composite sheet forming: A review," Composites - Part A: Applied Science and Manufacturing, vol. 33, no. 4, pp. 515-537, 2002.

2. C. Mack and H. Taylor, "The fitting of woven cloth to surfaces," Journal of the Textile Institute, vol. 47, pp. 477-488, 1956.

3. F. Van Der Weeën, "Algorithms for draping fabrics on doubly-curved surfaces," International Journal for Numerical Methods in Engineering, vol. 31, no. 7, pp. 1415-1426, 1991.

4. A. Cherouat and H. Bourouchaki, "Numerical tools for composite woven fabric preforming," Advances in Materials Science and Engineering, vol. 2013, 2013.

5. D.-J. Lukaszewicz, C. Ward and K. Potter, "The engineering aspects of automated prepreg layup: History, present and future," Composites Part B: Engineering, vol. 43, pp. 997-1009, 2012.

6. B. Tatting and Z. Gürdal, "Design and manufacure of elastically tailored tow placed plates," NASA/CR--2002-211919, 2002.

7. Z. Gürdal, B. Tatting and C. Wu, "Variable stiffness composite panels: Effects of stiffness variation on the in-plane and buckling response," Composites Part A: Applied Science and Manufacturing, vol. 39, no. 5, pp. 911-922, 2008.

8. A. Blom, B. Tatting, J. Hol and Z. Gürdal, "Fiber path definitions for elastically tailored conical shells," Composites Part B: Engineering, vol. 40, no. 1, pp. 77-84, 2009.

9. C. Waldhart, "Analysis of Tow-Placed, Variable-Stiffness Laminates," 1996.

10. B. Shirinzadeh, G. Cassidy, D. Oetomo, G. Alici and M. Ang, "Trajectory generation for opencontoured structures in robotic fibre placement," Robotics and Computer-Integrated Manufacturing, vol. 23, pp. 380-394, 2007.

11. M. Bruyneel and S. Zein, "A modified Fast Marching Method for defining fiber placement trajectories over meshes," Computers and Structures, vol. 125, pp. 45-52, 2013.

12. M. Bruyneel and S. Zein. "A new strategy avoiding gaps and overlaps in the simulation of fiber placement trajectories", International Conference on Composite Structures - ICCS16, June 28-30, 2011, Porto, Portugal.

13. M. Bruyneel, J.C. Craveur, P. Gourmelen. "Optimisation des structures mécaniques: methodes numériques et éléments finis", Dunod, Paris, 2014.

14. C.J. Brampton and H.A. Kim. "Optimization of tow steerd fiber orientation using the level set method", $10^{\text {th }}$ World Congress on Structural and Multidisciplinary Optimization, May 19-24, 2013, Orlando, Florida, USA.

15. J. Sethian, Level Set Methods and Fast Marching Methods, Cambridge University Press, 1999.

16. Y. Radovcic and A. Remouchamps, "BOSS QUATTRO: an open system for parametric design," Structural and Multidisciplinary Optimization, vol. 23, no. 2, pp. 140-152, 2002.

17. B. Colson, M. Bruyneel, S. Grihon, C. Raick and A. Remouchamps, "Colson B., Bruyneel M., Grihon S., Raick C. and Remouchamps A. (2010). "Optimisation methods for advanced design of aircraft panels: a comparison", Optimisation \& Engineering, 11(4), pp. 583-596.

18. M. Hyer and R. Charette, "Use of curvilinear fiber format in composite structure design," AIAA Journal, vol. 29, no. 6, pp. 1011-1015, 1991.

19. A. Pressley. Elementary differential geometry, London, Springer-Verlag, 2012.

20. J. Mitchell, D. Mount and C. Papadimitriou. "The discrete geodesic problem", SIAM Journal of Computing, 16 (4), pp. 647-668, 1987.

21. D. Coeurjolly, S. Miguet and L. Tougne. "Discrete curvature based on osculating circle estimation", in Visual Form 2001, Lecture Notes in Computer Science, vol. 2059, pp. 303-312, 2001. 